\title{
Non-mammary metastases to the breast and axilla: a study of 85 cases
}

\author{
Deborah F DeLair, Adriana D Corben, Jeffrey P Catalano, Christina E Vallejo, Edi Brogi and \\ Lee K Tan
}

Department of Pathology, Memorial Sloan-Kettering Cancer Center, New York, NY, USA

\begin{abstract}
Non-mammary metastases to the breast and axilla are rare occurrences. However, they are important diagnostic considerations as their treatment and prognosis differ significantly from primary breast cancer. Between 1990 and 2010, we identified a total of 85 patients, 72 women and 13 men, with non-mammary malignancies involving the breast, axilla, or both. The tumor types consisted of carcinoma (58\%), melanoma (22\%) and sarcoma (20\%). Ovary was the most common site of origin for carcinoma, and metastatic high-grade ovarian serous carcinoma was most frequently misdiagnosed as a primary breast carcinoma. Melanoma was the single most common non-carcinomatous tumor type to involve the breast and/or axilla, and uterine leiomyosarcoma was the most common type of sarcoma. Most patients $(77 \%)$ had other metastases at the time of diagnosis of the tumor, but in $11 \%$ the breast or axillary lesion was the first presentation. Without a clinical history, non-mammary metastases were difficult to diagnose because the majority of cases presented with a solitary nodule and lacked pathognomonic pathologic features. There were, however, certain recurrent histological findings identified, such as the often relatively well-circumscribed growth pattern of the metastatic lesion surrounded by a fibrous pseudocapsule, and the absence of an in situ carcinoma. Overall, these patients had poor survival; $96 \%$ of patients with follow-up available are dead of disease, with a median survival of 15 months after the diagnosis of the breast or axillary lesion. This finding emphasizes the need to accurately identify these tumors as metastases in order to avoid unnecessary procedures and treatments in these patients. Modern Pathology (2013) 26, 343-349; doi:10.1038/modpathol.2012.191; published online 23 November 2012
\end{abstract}

Keywords: axilla; breast; extramammary

Metastases to the breast and axilla are rare and account for approximately $2 \%$ of all mammary malignancies. ${ }^{1,2}$ The most common metastatic lesion to involve the breast is a metastasis from a contralateral mammary cancer. $^{1,2}$ If hematologic malignancies are also excluded, the number of non-mammary metastases drops to well below $1 \% .^{1,3}$ Owing to the frequency of primary breast cancers and the rarity of non-mammary tumors involving the breast, a newly discovered lesion in the breast or axilla is usually presumed to be a primary or metastatic breast carcinoma. However, the recognition of non-mammary metastases to the breast and axilla is very important, as both the treatment and prognosis differ significantly.

Correspondence: Dr DF DeLair, MD, Department of Pathology, Memorial Sloan-Kettering Cancer Center, 1275 York Avenue, New York, NY10065, USA.

E-mail: gynbreast@mskcc.org

Received 13 June 2012; revised 18 September 2012; accepted 19 September 2012; published online 23 November 2012

\section{Materials and methods}

After obtaining approval by our Institutional Review Board, we searched the pathology database of Memorial Sloan-Kettering Cancer Center between the years 1990-2010 for patients with non-mammary metastases to the breast and axilla. Hematologic malignancies were excluded, as they are systemic diseases. The medical records and pathology slides, when available, were reviewed and the clinicopathologic characteristics were recorded. Patient age, gender, primary tumor site, the presence of other metastases, laterality, number of lesions, interval from primary tumor diagnosis to breast/ axillary metastasis, imaging studies and outcome data were noted. Pathological parameters recorded included tumor histology and size, and certain histological features, including growth pattern (circumscribed versus infiltrative), the presence of a pseudocapsule, the nature of the surrounding tissue, and whether in situ carcinoma, calcifications, or lymphovascular invasion, were identified. The use of immunohistochemistry to aid in the diagnosis was also noted. 


\section{Results}

Our search identified a total of 85 patients (72 women, 13 men) with metastatic lesions in the breast, axilla or both. The clinical features are summarized in Table 1 . The median patient age was 54 years (range, 15-83). The majority of patients were women $(85 \%, 72 / 85)$. Breast only involvement was present in $78 \%(66 / 85)$ of the patients, axilla only in $8 \%(7 / 85)$ and both in $14 \%(12 / 85)$. The breast or axillary lesion was the first presentation in $11 \%(9 / 85)$ of patients. The tumors were frequently unilateral $(88 \%, 75 / 85)$ and solitary $(75 \%, 64 / 85)$. The median tumor size was $1.68 \mathrm{~cm}$ (range, 0.5$18 \mathrm{~cm}$ ). Among patients who had the primary tumor diagnosed first, the median interval from the primary to the metastatic breast/axillary lesion was 4.5 years (range, synchronous-16 years). The large majority of patients $(77 \%, 66 / 85)$ had other metastases at the time of the breast/axillary lesion.

Imaging studies were available for 66 patients and included mammogram, CT scan, ultrasound and MRI. Metastasis was favored in $42 \%$ (28/66) of cases, while primary breast carcinoma was suspected in $15 \%(10 / 66)$. In $27 \%(18 / 66)$ of the patients, the differential diagnosis included primary versus metastasis. In $16 \%$ of cases, either a benign $(8 \%, 5 / 66)$ or an indeterminate $(8 \%, 5 / 66)$ lesion was considered.

Carcinoma was the most common tumor type identified $(58 \%, 49 / 85)$, followed by melanoma $(21 \%, 18 / 85)$ and sarcoma $(21 \%, 18 / 85)$. The different types of non-mammary metastases are summarized in Tables 2-4. Among patients with carcinomas (Table 2), the most common primary site was the gynecologic tract $(39 \%, 19 / 49)$, with ovary as the single most frequent primary source $(29 \%, 14 / 49)$. Lung was the next most common primary site $(22 \%$, $11 / 49)$, followed by the gastrointestinal tract $(13 \%$, $7 / 49)$. Other primary sites included the genitourinary tract $(10 \%, 5 / 49)$, head and neck $(8 \%, 4 / 49)$, and, rarely, skin (Merkel cell carcinoma (4\%, 2/49)).

Melanoma was the single most common noncarcinomatous metastatic tumor and represented $22 \%(18 / 85)$ of all cases (Table 3$)$. The majority of the melanomas arose in cutaneous sites; however, three of the cases were ocular in origin. In patients with axillary involvement only, melanoma was the most common type of metastasis.

Among the sarcomas, uterine leiomyosarcoma was the most frequent $(28 \%, 5 / 18)$, followed by rhabdomyosarcoma $(17 \%, 3 / 18)$, liposarcoma (11\%, $2 / 18)$, and non-uterine leiomyosarcoma (11\%, 2/18). Other sarcomas identified included Ewing sarcoma, pleomorphic sarcoma (malignant fibrous histiocytoma), synovial sarcoma, angiosarcoma, dendritic cell sarcoma, and myofibrosarcoma.

Glass slides were available for review in 53 out of $85(62 \%)$ cases. These slides included 28 core biopsies and 30 excisions ( 5 cases with both cores and excisions). Seven cases involved lymph nodes
Table 1 Clinical features

\begin{tabular}{ll}
\hline Clinical feature & Result \\
\hline Median age (years) & 54 (range 15-83) \\
Female/male & $72: 13(85 \%: 15 \%)$ \\
Unilateral/bilateral & $75: 10(88 \%: 12 \%)$ \\
Solitary/multiple & $64: 21(75 \%: 15 \%)$ \\
Median tumor size (cm) & $1.68($ range $0.5-18)$ \\
Breast/axillary lesion diagnosed first & $9 / 85(11 \%)$ \\
Median interval from diagnosis of primary & $4.5($ range \\
to breast/axillary metastasis (years) & synchronous-16) \\
Axillary metastases only & $7 / 85(8 \%)$ \\
Metastasis favored by imaging & $28 / 85(42 \%)$ \\
Other metastases at time of diagnosis of & $66 / 85(77 \%)$ \\
breast/axillary lesion & 15 (range <1-83) \\
Median survival (months) & \\
\hline
\end{tabular}

only ( 6 axillary and 1 intramammary). The remaining 46 cases all involved the breast. Mammary lymphatic only involvement, without stromal invasion, occurred in $7 \%(3 / 46)$ of the cases. Certain recurrent histological features were identified, including a well-circumscribed nodule $(74 \%, 34 / 46)$, often surrounded by a fibrous pseudocapsule (52\%, 24/46) with varying thickness (Figure 1) and absence of an in situ mammary carcinoma. Interestingly, in the great majority of the tumors, lymphovascular invasion was notably absent $(87 \%, 40 / 46)$. Tumoral calcifications were observed in non-mammary metastases of serous carcinoma type that were psammomatous in nature $(75 \%, 6 / 8)$ (Figure 2), including two cases that involved lymph nodes.

Immunohistochemistry was used in the initial work-up of $38(45 \%, 38 / 85)$ cases. In all of these cases, the primary tumors were known, except for one case in which a patient had a choriocarcinoma that had yet to be detected. Melanoma was the most frequent tumor stained (9/85), followed by lung (6/ 85). The most frequently used antibodies included ER, PR, AE1:AE3, S100, and TTF-1. Diagnostic errors occurred in 10 cases $(12 \%, 10 / 85)$, the majority of these being ovarian high-grade serous carcinoma $(60 \%, 6 / 10)$ (Figure 3). In three of the patients with ovarian cancer who were misdiagnosed as a primary breast carcinoma, the biopsy was initially misinterpreted at an outside institution. After a full clinical work-up was performed at MSKCC, the ovarian primaries were discovered and this information was then communicated to the pathologist and the diagnoses were subsequently amended. In the cases of the remaining three patients with ovarian cancer, the patients were initially treated at another institution and the history of the ovarian primaries was not immediately available. After this history was revealed, the previous material was obtained and compared to the breast/axillary lesion, resulting in a correct diagnosis. The patients with melanoma and lung primaries were misdiagnosed, again, due to the failure of the clinician to communicate the history of a prior malignancy. After this information was relayed to the pathologist, the diagnoses were subsequently 
Table 2 Carcinomas

\begin{tabular}{|c|c|}
\hline Tumor site/type $(n=49)$ & $\begin{array}{l}\text { No. of cases } /(\% \text { of } \\
\text { carcinomas })\end{array}$ \\
\hline \multicolumn{2}{|l|}{ Ovary $(\mathrm{n}=14)$} \\
\hline High-grade serous carcinoma & $10(21)$ \\
\hline Low-grade serous carcinoma & $3(6)$ \\
\hline Clear cell carcinoma & $1(2)$ \\
\hline \multicolumn{2}{|l|}{ Lung $(\mathrm{n}=11)$} \\
\hline Adenocarcinoma & $4(8)$ \\
\hline Large cell neuroendocrine & $3(6)$ \\
\hline Poorly differentiated carcinoma & $2(4)$ \\
\hline Small cell carcinoma & $1(2)$ \\
\hline 'Large’ cell carcinoma & $1(2)$ \\
\hline \multicolumn{2}{|l|}{ Gastrointestinal tract $(\mathrm{n}=7)$} \\
\hline Colonic adenocarcinoma & $3(6)$ \\
\hline Pancreatic adenocarcinoma & $2(4)$ \\
\hline Carcinoid (colon) & $1(2)$ \\
\hline Carcinoid (liver) & $1(2)$ \\
\hline \multicolumn{2}{|l|}{ Genitourinary tract $(\mathrm{n}=5)$} \\
\hline Urothelial carcinoma (bladder) & $2(4)$ \\
\hline Renal cell carcinoma & $2(4)$ \\
\hline Prostatic adenocarcinoma & $1(2)$ \\
\hline \multicolumn{2}{|c|}{ Gynecologic tract (excluding ovary) $(\mathrm{n}=5)$} \\
\hline $\begin{array}{l}\text { Endometrioid adenocarcinoma } \\
\text { (endometrial) }\end{array}$ & $1(2)$ \\
\hline Combined endometrioid/small cell & $1(2)$ \\
\hline \multicolumn{2}{|l|}{ carcinoma (endometrium) } \\
\hline $\begin{array}{l}\text { Undifferentiated carcinoma } \\
\text { (endometrium) }\end{array}$ & $1(2)$ \\
\hline Choriocarcinoma & $1(2)$ \\
\hline Small cell carcinoma (cervix) & $1(2)$ \\
\hline \multicolumn{2}{|l|}{ Thyroid $(\mathrm{n}=2)$} \\
\hline Papillary thyroid carcinoma & $2(4)$ \\
\hline Medullary thyroid carcinoma & $1(2)$ \\
\hline \multicolumn{2}{|l|}{$\operatorname{Skin}(\mathrm{n}=2)$} \\
\hline Merkel cell carcinoma & $2(4)$ \\
\hline \multicolumn{2}{|l|}{ Submandibular gland $(\mathrm{n}=1)$} \\
\hline Adenoid cystic carcinoma & $1(2)$ \\
\hline \multicolumn{2}{|l|}{ Tongue $(\mathrm{n}=1)$} \\
\hline Squamous cell carcinoma & $1(2)$ \\
\hline
\end{tabular}

changed. In the remaining two cases, the primary tumors, medullary thyroid carcinoma (Figure 4) and hepatic carcinoid tumor (Figure 5) had yet to be discovered. After a full clinical work-up, the thyroid and liver lesions were found and compared to the metastatic tumors. In addition, immunohistochemical stains were performed on the metastases, which helped to render the correct diagnoses.

Males in this series represented $15 \%$ of the cases. The breast was involved in all cases, with or without axillary involvement. The most common metastatic tumor was melanoma, followed by lung, renal cell carcinoma, Merkel cell carcinoma, prostate (Figure 6), and papillary thyroid carcinoma. Two patients presented with gynecomastia and a primary breast carcinoma was suspected in both.

Current follow-up data were available for 55 of the patients. The vast majority of patients have died of
Table 3 Melanomas

\begin{tabular}{lc} 
Site $(\mathrm{n}=18)$ & No. of cases (\% of melanomas) \\
\hline Cutaneous & $15(87)$ \\
Ocular & $3(13)$ \\
\hline
\end{tabular}

Table 4 Sarcomas

Tumor type $(\mathrm{n}=18)$ No. of cases (\% of sarcomas)

Uterine leiomyosarcoma $5(28)$

Rhabdomyosarcoma

Liposarcoma

Non-uterine leiomyosarcoma

Ewing sarcoma

Pleomorphic sarcoma (MFH)

Angiosarcoma

Synovial sarcoma

Dendritic cell sarcoma

3 (17)

$2(11)$

$2(11)$

$1(5.5)$

$1(5.5)$

$1(5.5)$

$1(5.5)$

Myofibrosarcoma

disease $(96 \%, 52 / 55)$ and had a median survival of 15 months following the diagnosis of the breast or axillary lesion. Two of the remaining three patients are alive with disease, while the third is currently free of disease, with an average follow-up of 46 months.

\section{Discussion}

Non-hematologic metastases to the breast and axilla are rare occurrences. They usually developed in the fifth or sixth decade and the patients most often had a prior history of a malignant tumor with documented metastatic spread. ${ }^{1,2,4-6}$ A wide variety of malignancies from many different sites have been reported. The most common reported sites of origin in the literature include the lung, skin, stomach, and ovary. ${ }^{1,2,4-6}$

In this series, ovary was the most common primary site among all of the carcinomas. In keeping with other reported series, ${ }^{7-9}$ our study found that high-grade serous carcinoma was the most frequent ovarian carcinoma type to metastasize to the breast or axilla. This tumor was also the type most often misdiagnosed initially due to overlapping morphologic and immunohistochemical features with a primary breast cancer. Both tumor types can have a wide range of histologic appearances, including papillary, glandular, and solid architecture with necrosis and high-grade cytology; it can be extremely difficult to differentiate the two without knowledge of a prior ovarian primary. In some instances, even when a primary ovarian carcinoma is known, it may still be a diagnostic challenge morphologically. Immunohistochemical studies can be helpful in problematic cases. ER and PR are frequently expressed in both breast and ovarian carcinomas and are therefore of limited value. PAX8, 


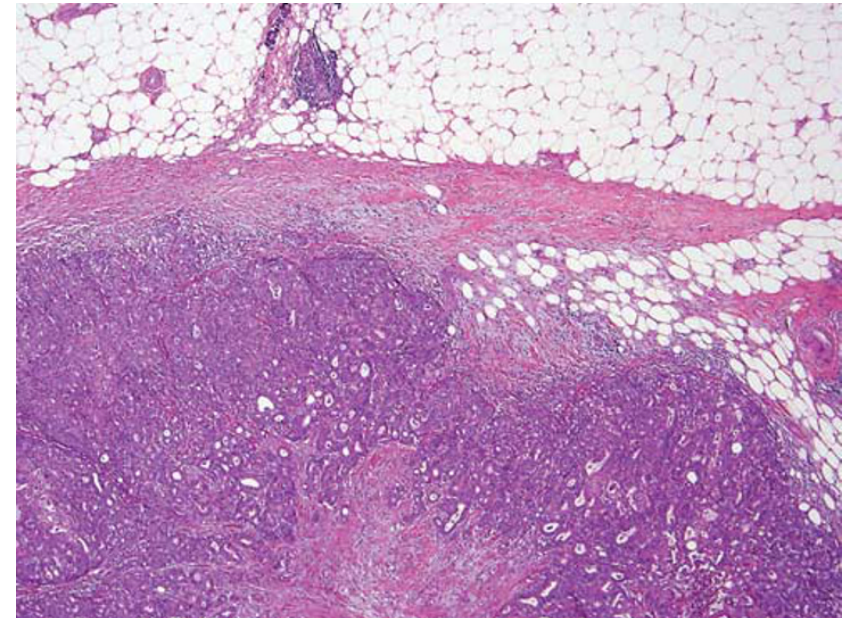

Figure 1 Metastatic colorectal adenocarcinoma to the breast. Note the relatively well-circumscribed lesion surrounded by a fibrous pseudocapsule.

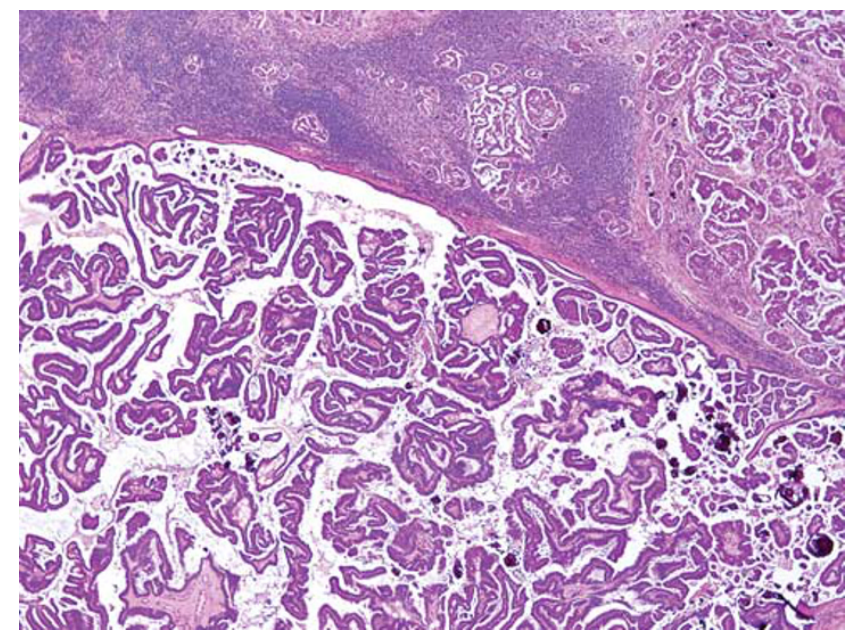

Figure 2 Low-grade ovarian serous carcinoma metastatic to an intramammary lymph node. Serous carcinomas were the only metastatic tumors to be associated with calcifications.

on the other hand, has been shown to be positive in nearly all gynecologic cancers and, to date, is negative in breast cancers. ${ }^{10,11}$ In one study of PAX8 expression in ovarian and breast carcinoma, the marker was immunoreactive in $87 \%(108 / 124)$ of the ovarian carcinomas studied. The ovarian tumors positive for PAX8 included serous, endometrioid, and clear cell, while mucinous carcinoma was usually negative. If ovarian mucinous carcinomas were excluded, PAX 8 was positive in $96 \%$ of the primary ovarian carcinomas. None of the 243 cases of breast carcinoma studied were found to be PAX8 positive. ${ }^{11} \mathrm{WT} 1$ is another marker that may be useful in this setting. It has been shown to be a rather sensitive marker in ovarian serous carcinoma as it is positive in up to $85 \%$ of the cases, ${ }^{12}$ while rarely positive in breast cancer $(2.1 \%$ in one report). ${ }^{11}$ There are a few exceptions, however.

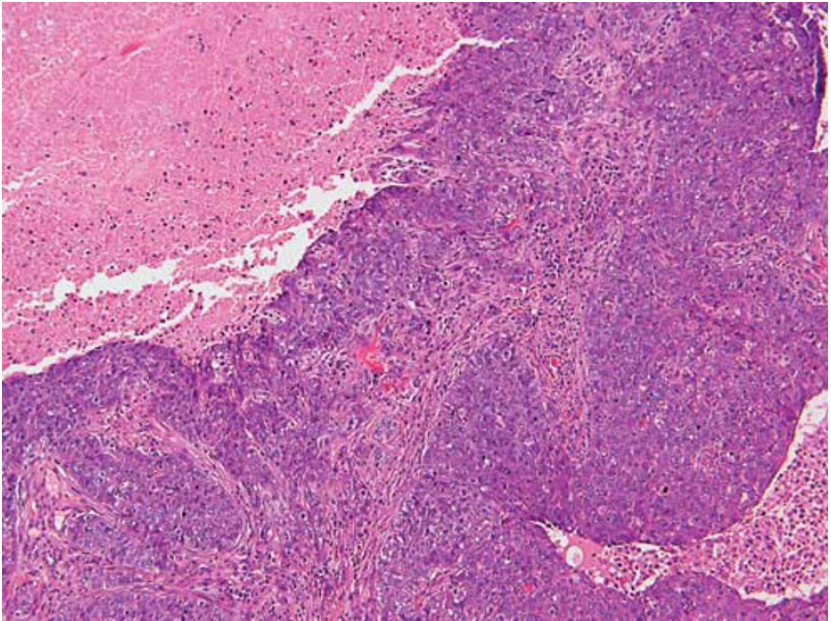

Figure 3 High-grade ovarian serous carcinoma metastatic to the breast. The tumor has overlapping morphologic features with primary breast cancer with medullary or atypical medullary features including a solid growth pattern, central necrosis, a host inflammatory response and marked cytologic atypia. Ovarian high-grade serous carcinoma was the most commonly misdiagnosed tumor in this series.

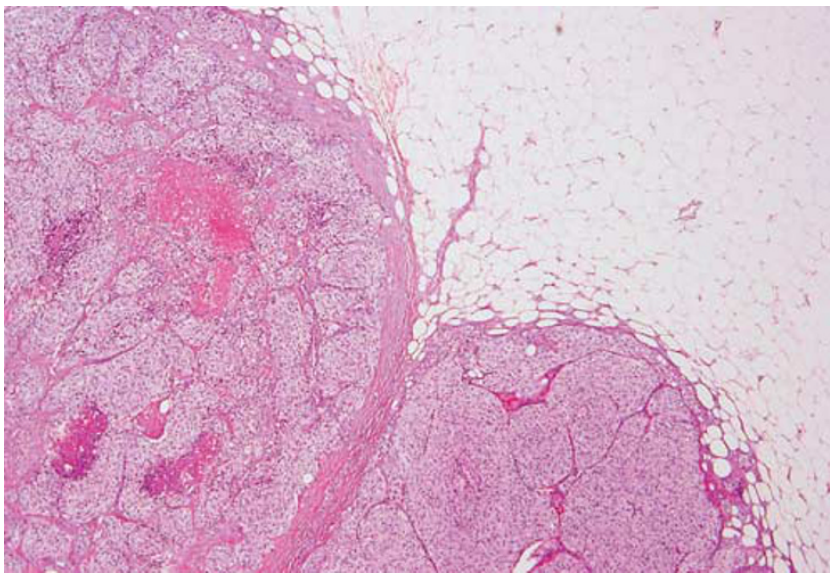

Figure 4 Metastatic medullary thyroid carcinoma to the breast. This tumor was initially misinterpreted to be a primary breast cancer.

Domfeh et $a l^{13}$ found WT1 expression in 64\% (21/ 33 ) of pure and $33 \%(11 / 33)$ of mixed mucinous breast carcinomas with either ductal (no special type) or micropapillary components; however, the WT1 staining was usually weak and focal in most of the positive breast tumors.

As in our series, the most commonly reported gynecologic cancer to metastasize to the breast is serous carcinoma. ${ }^{7-9}$ Other unusual gynecologic tumors reported to have metastasized to the breast included endometrial stromal sarcoma, ${ }^{14}$ ovarian clear cell carcinoma, ${ }^{7}$ ovarian granulosa cell tumor ${ }^{15}$ ovarian dysgerminoma ${ }^{16}$ and choriocarcinoma. ${ }^{5}$ The current study included one ovarian clear cell carcinoma and one choriocarcinoma.

Another gynecologic tumor involving the breast was uterine leiomyosarcoma, comprising $5-6 \%$ of cases. In addition to gynecologic leiomyosarcomas, 


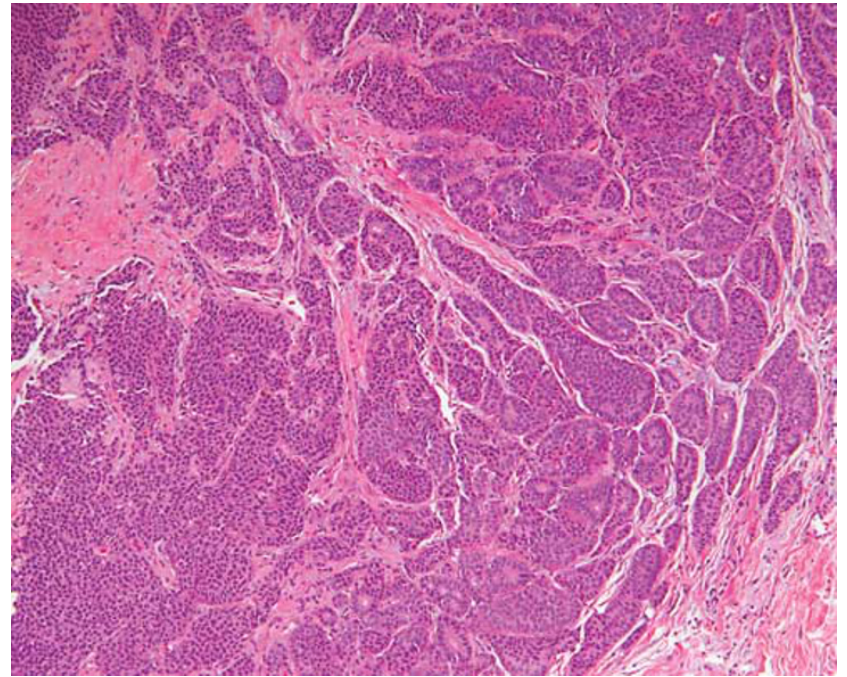

Figure 5 Metastatic hepatic carcinoid tumor to the breast. This tumor was initially misinterpreted to be a primary breast cancer.

two extrauterine leiomyosarcomas were identified, one from the retroperitoneum and one from the leg. Several other cases of uterine leiomyosarcoma metastatic to the breast have also been reported. ${ }^{17-20}$ Primary leiomyosarcoma of the breast is exceedingly rare, with only 44 cases reported in the literature, ${ }^{21}$ but a metastatic leiomyosarcoma to the breast is even more unusual.

The single most common non-carcinomatous lesion encountered in this study was melanoma $(22 \%)$. This is in accordance with previous large series, if hematologic malignancies are excluded., ${ }^{1,2,6}$ The majority of the reported cases in the literature have originated from cutaneous sites; ${ }^{1,6,22,23}$ however, two series included melanomas arising from the eye. ${ }^{1,23}$ In a report of 27 melanomas involving the breast, $74 \%$ arose from cutaneous sites, while $11 \%$ were ocular melanomas; these rates are similar to those found in our series. ${ }^{23}$ One of the six melanomas reported in the series by Georgiannos et $a l^{1}$ was also an ocular melanoma $(17 \%)$. This is unusual as only $4-5 \%$ of melanomas arise from noncutaneous sites. ${ }^{24}$ Melanoma was also the most common histology to involve the axilla and the most common metastatic lesion found in men. Owing to its wide range of morphologic appearances, it may be easily mistaken for a primary breast cancer. Immunohistochemical stains for cytokeratins and melanoma markers (S100, HMB45, Melan-A) can easily differentiate the two.

In the pediatric population, the most common tumor metastatic to the breast in the pediatric population is rhabdomyosarcoma; this tumor seems to occur more frequently in adolescent girls than in boys, as almost all of the reported cases have been in the former. ${ }^{25-31}$ In two different series of metastatic tumors to the breast, each reported four adolescent girls with metastatic rhabdomyosarcoma involving the breast. The alveolar subtype has been reported to

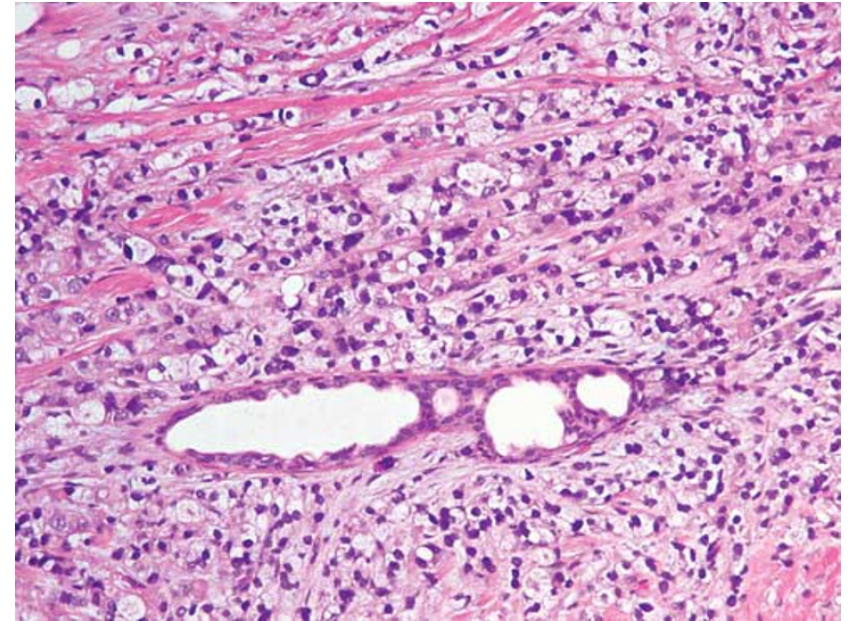

Figure 6 Metastatic prostatic adenocarcinoma metastatic to the breast. Note the entrapped benign duct. This is unusual as most tumors were surrounded by adipose tissue and did not infiltrate normal breast parenchyma. This tumor was diffusely positive with PSA by immunohistochemistry.

occur much more commonly than embryonal, which is well known to be much more aggressive. Our series included three patients with metastatic alveolar rhabdomyosarcoma: two in adolescent girls 15 and 18 years old, and the other in a 28year-old woman.

In this series, the failure of the pathologist to recognize the metastatic nature of the lesion resulted most often because of the absence of a prior cancer history provided by the clinician at the time of initial interpretation. Six of these tumors were highgrade serous carcinoma and were misdiagnosed because of the overlapping morphological and immunohistochemical features as described previously. The other tumors (lung adenocarcinoma, liver carcinoid, medullary thyroid carcinoma, and melanoma) all had appearances that may be compatible with a primary breast cancer. Tumors that appear to be relatively well-differentiated, however, would be expected to express estrogen and progesterone receptors if they were indeed primary mammary carcinomas. Such a finding should prompt the pathologist to investigate the clinical history more thoroughly in order to rule out or suggest the possibility of a metastatic lesion. Compounding the problem is the observation in our study that $75 \%$ of metastases presented as solitary lesions, as opposed to multiple metastatic lesions commonly observed in other organs such as liver and lung. The implementation of electronic medical records in our institution has allowed the pathologist easy access to the patient's history. Despite this, our clinicians are urged to always provide a complete history, as records and procedures from outside institutions are not always immediately available.

Imaging studies are not always helpful in arriving at the correct diagnosis, as $24 \%$ of the cases that had imaging studies available were mistakenly inter- 
preted as a primary breast cancer or a benign lesion. This finding has also been previously reported in the literature. ${ }^{30}$ Similarly to the above, a contributing factor to these interpretations is the fact that the majority of these tumors presented as a solitary lesion in the breast and/or axilla.

Several recurrent histological features were identified in metastatic tumors to the breast. These included a well-circumscribed lesion, sharply demarcated from the adjacent tissue and the absence of in situ carcinoma and calcifications. ${ }^{2,5,30,32}$ The exception to this is serous ovarian carcinoma, which very frequently has psammomatous calcifications. ${ }^{8,9}$ These features contrast with primary breast carcinoma, as it is usually an ill-defined lesion, infiltrates breast parenchyma, and often contains in situ carcinoma and calcifications (non-psammomatous). While most studies have found similar results, in the series by Lee et $a l,{ }^{4}$ calcifications were identified in one hepatocellular carcinoma and two gastric carcinomas metastatic to the breast.

Metastases to the breast and or axilla represent an important diagnostic consideration as these patients have a poor prognosis. In a series of 169 patients with metastatic tumors to the breast, the median survival from the time of diagnosis was 10 months, with a range of less than a month to 192 months. ${ }^{6}$ In our series, the patients had a median survival of 15 months (range, less than 1 month to 83 months). Other studies have shown similar outcomes. ${ }^{2-4}$ This poor survival is due to the fact that at the time of the discovery of the breast lesion, the majority of the patients already had widely metastatic disease. Thus, the finding of a metastatic tumor in the breast usually portends a poor prognosis, as these lesions rarely present without the presence of other metastases. Arriving at the correct diagnosis is crucial so as to avoid unnecessary procedures and treatments in these patients.

\section{Disclosure/conflict of interest}

The authors declare no conflict of interest.

\section{References}

1 Georgiannos SN, Chin J, Goode AW, et al. Secondary neoplasms of the breast: a survey of the 20th Century. Cancer 2001;92:2259-2266.

2 Hajdu SI, Urban JA. Cancers metastatic to the breast. Cancer 1972;29:1691-1696.

3 Chaignaud B, Hall TJ, Powers C, et al. Diagnosis and natural history of extramammary tumors metastatic to the breast. J Am Coll Surg 1994;179:49-53.

4 Lee SK, Kim WW, Kim SH, et al. Characteristics of metastasis in the breast from extramammary malignancies. J Surg Oncol 2010;101:137-140.
5 McIntosh IH, Hooper AA, Millis RR, et al. Metastatic carcinoma within the breast. Clin Oncol 1976;2: 393-401.

6 Williams SA, Ehlers RA 2nd, Hunt KK, et al. Metastases to the breast from nonbreast solid neoplasms: presentation and determinants of survival. Cancer 2007;110:731-737.

7 Moore DH, Wilson DK, Hurteau JA, et al. Gynecologic cancers metastatic to the breast. J Am Coll Surg 1998; 187:178-181.

8 Recine MA, Deavers MT, Middleton LP, et al. Serous carcinoma of the ovary and peritoneum with metastases to the breast and axillary lymph nodes: a potential pitfall. Am J Surg Pathol 2004;28:p 1646-1651.

9 Yamasaki H, Saw D, Zdanowitz J, et al. Ovarian carcinoma metastasis to the breast case report and review of the literature. Am J Surg Pathol 1993;17: 193-197.

10 Laury AR, Perets R, Piao $\mathrm{H}$, et al. A comprehensive analysis of PAX8 expression in human epithelial tumors. Am J Surg Pathol 2011;35:816-826.

11 Nonaka D, Chiriboga L, Soslow RA. Expression of pax8 as a useful marker in distinguishing ovarian carcinomas from mammary carcinomas. Am J Surg Pathol 2008;32:1566-1571.

12 Acs G, Pasha T, Zhang PJ. WT1 is differentially expressed in serous, endometrioid, clear cell, and mucinous carcinomas of the peritoneum, fallopian tube, ovary, and endometrium. Int J Gynecol Pathol 2004;23:110-118.

13 Domfeh AB, Carley AL, Striebel JM, et al. WT1 immunoreactivity in breast carcinoma: selective expression in pure and mixed mucinous subtypes. Mod Pathol 2008;21:1217-1223.

14 Gunhan-Bilgen I, Memis A, Ustun EE. Metastatic intramammary lymph nodes: mammographic and ultrasonographic features. Eur J Radiol 2001;40:24-29.

15 Harwood TR. Metastatic carcinoma to the breast. JAMA 1971;218:97.

16 Kattan J, Droz JP, Charpentier P, et al. Ovarian dysgerminoma metastatic to the breast. Gynecol Oncol 1992;46:104-106.

17 David O, Gattuso P, Razan W, et al. Unusual cases of metastases to the breast. A report of 17 cases diagnosed by fine needle aspiration. Acta Cytol 2002;46:377-385.

18 Lin $\mathrm{CH}$, Yeh CN, Chen MF. Breast metastasis from uterine leiomyosarcoma: a case report. Arch Gynecol Obstet 2003;267:233-235.

19 Pappa L, Zagorianakou N, Kitsiou E, et al. Breast metastasis from uterine leiomyosarcoma diagnosed by fine-needle aspiration: a case report. Acta Cytol 2008;52:485-489.

20 Sibartie S, Larkin JO, Lee G, et al. Metastatic uterine leiomyosarcoma presenting as a breast lump. Ir J Med Sci 2011;180:889-891.

21 Fujita N, Kimura R, Yamamura J, et al. Leiomyosarcoma of the breast: a case report and review of the literature about therapeutic management. Breast 2011; 20:389-393.

22 Loffeld A, Marsden JR. Management of melanoma metastasis to the breast: case series and review of the literature. Br J Dermatol 2005;152:1206-1210.

23 Ravdel L, Robinson WA, Lewis K, et al. Metastatic melanoma in the breast: a report of 27 cases. J Surg Oncol 2006;94:101-104.

24 Hussein MR. Extracutaneous malignant melanomas. Cancer Invest 2008;26:516-534. 
25 Chateil JF, Arboucalot F, Pérel Y, et al. Breast metastases in adolescent girls: US findings. Pediatr Radiol 1998;28:832-835.

26 Kwan WH, Choi PH, Li CK, et al. Breast metastasis in adolescents with alveolar rhabdomyosarcoma of the extremities: report of two cases. Pediatr Hematol Oncol 1996;13:277-285.

27 Oksuzoglu B, Abali H, Güler N, et al. Metastasis to the breast from nonmammarian solid neoplasms: a report of five cases. Med Oncol 2003;20:295-300.

28 Shukla R, Pooja B, Radhika S, et al. Fine-needle aspiration cytology of extramammary neoplasms metastatic to the breast. Diagn Cytopathol 2005;32: 193-197.
29 Sneige N, Zachariah S, Fanning TV, et al. Fine-needle aspiration cytology of metastatic neoplasms in the breast. Am J Clin Pathol 1989;92:27-35.

30 Vergier B, Trojani M, de Mascarel I, et al. Metastases to the breast: differential diagnosis from primary breast carcinoma. J Surg Oncol 1991;48:112-116.

31 Vizcaino I, Trojani M, de Mascarel I, et al. Metastasis to the breast from extramammary malignancies: a report of four cases and a review of literature. Eur Radiol 2001;11:1659-1665.

32 Ribeiro-Silva A, Mendes CF, Costa IS, et al. Metastases to the breast from extramammary malignancies: a clinicopathologic study of 12 cases. Pol J Pathol 2006; 57:161-165. 